\title{
An update on intimate partner violence and mental health
}

\author{
Giulia RIOLI ${ }^{1}$, Chiara SGARBI ${ }^{2}$, Valentina MORETTI ${ }^{3}$, Arianna SINISI ${ }^{1}$, \\ Laura DE FAZIO ${ }^{2}$, Nina GIAMBALVO ${ }^{1}$, Silvia FERRARI ${ }^{1}$, Gian M. GALEAZZI 1 *
}

${ }^{1}$ Section of Psychiatry, Department of Diagnostic-Clinical Medicine and Public Health, University of Modena and Reggio Emilia, Modena, Italy; ${ }^{2}$ Department of Law, University of Modena and Reggio Emilia, Modena, Italy; ${ }^{3}$ Department of Mental Health and Substance Use Disorders, Reggio Emilia, Italy

*Corresponding author: Gian M. Galeazzi, Section of Psychiatry, Department of Diagnostic-Clinical Medicine and Public Health, University of Modena and Reggio Emilia, via del Pozzo 71, 41124 Modena, Italy. E-mail: gianmaria.galeazzi@unimore.it

\section{A B S T R A C T}

INTRODUCTION: Intimate partner violence (IPV) is a problem worldwide and a human rights issue. The present paper summarizes recent literature on the relationship between IPV and mental health, exploring risk factors for psychiatric patients to be victims and/or perpetrators of violent behavior in intimate relationships.

EVIDENCE ACQUISITION: A search of PubMed, PsychINFO and Cochrane Library databases was performed, looking for papers concerning IPV and psychiatry published between 2011 and 2016. Papers were retrieved and reviewed according to PRISMA statement guidelines. Only research papers with sample sizes $>50$ adult subjects were included, leading to a first selection of 1486 records. Titles and abstracts were independently screened by three pairs of researchers, following predefined criteria agreed by the authors, yielding 93 articles for review.

EVIDENCE SYNTHESIS: Mental disorders, especially posttraumatic stress disorder (PTSD) and depression, are associated with risk for perpetrating IPV. Confounding factors of this association are substance use/dependence, childhood adversity, previous IPV perpetration. Psychiatric patients are at high risk of becoming victims of IPV, but specific risk factors for victimization in this population have been poorly investigated. Rates of PTSD, depression, anxiety and substance use disorders are higher among IPV victims than in the general population. Interpersonal variables and social factors play a key role both in the onset and in the recovery from mental disorders in the aftermath of IPV.

CONCLUSIONS: Individuals suffering from a mental disorder are prone to be both victims and perpetrators of IPV. Further research efforts are needed to elucidate the risk factors for victimization, in order to devise effective risk management strategies.

(Cite this article as: Rioli G, Sgarbi C, Moretti V, Sinisi A, De Fazio L, Giambalvo N, et al. An update on intimate partner violence and mental health. Minerva Psichiatr 2017;58: DOI: 10.23736/S0391-1772.17.01943-4)

Key words: Intimate partner violence - Psychiatry - Mental health - Crime victims.

\section{Introduction}

I ntimate partner violence (IPV), as a pattern of aggressive behaviors perpetrated by a current or former intimate partner, is a global problem, constituting an important public health and human rights issue. ${ }^{1,2}$ It is a complex behavior, that can take many forms (physical, sexual, psychological and economic), historically conceived as a manifestation of gender inequality and male patriarchal stereo- types and values. ${ }^{3}$ Only recently have Western societies started focusing attention on IPV, shifting from considering it as a family private matter to a public one, and recognizing it as an expression of power, control, oppression and discrimination. ${ }^{4}$ Despite the social and cultural changes that have occurred over the last few years and the increase in equal opportunities policies, such intentional violation of victim dignity and personality integrity remains a widespread phenomenon, affecting an intoler- 
ably high number of individuals worldwide, regardless of age, economic status, educational level, ethnic background, sexual preference or religion. 4,5

Data published by the World Health Organization show the epidemic prevalence of IPV, as an overall $30 \%$ of all women worldwide have experienced physical or sexual violence by their intimate partner. For female homicide, the murderer is the victim's partner in $38 \%$ of cases. $^{2}$ Equally disturbing are the numbers reported by the United Nations, with a lifetime prevalence of IPV ranging from $6 \%$ to $64 \%$ for physical and/or sexual IPV and from $7 \%$ to $68 \%$ for emotional/psychological forms. ${ }^{5}$ The National Intimate Partner and Sexual Violence Survey reported that, in the USA, more than $37 \%$ of women have suffered physical violence, contact sexual violence and/or stalking by an intimate partner at least once in their lifetime, and that around $50 \%$ have endured psychological abuse. Over $27 \%$ of the victims reported a subsequent IPV-related impact on their lives. ${ }^{6}$ The European Institute for Gender Equality has monitored the phenomenon of IPV in $28 \mathrm{EU}$ member states, reporting a lifetime prevalence of partner physical violence ranging from $4 \%$ to $37 \%$. $^{7}$ Furthermore, the results of the European Union Agency for Fundamental Rights survey on violence against women indicate that $22 \%$ of women are victims of physical and/or sexual violence by a partner in their lifetime, $8 \%$ during the past year, with $43 \%$ reporting some forms of psychological aggression and 13\% economic victimization. ${ }^{8}$

While the phenomenon of IPV afflicts women as its main victims, it also affects the whole of society and demands international efforts to reduce its occurrence, by the development of appropriate intervention strategies and the promulgation of specific conventions and treaties. In particular, the 2011 Istanbul Convention highlighted the need for a concerted approach, involving national governments in ensuring they address discrimination and violence against women in the context of prevention, victim protection and perpetrator prosecution. ${ }^{9}$ The Convention has defined vio- lence in intimate or domestic context as "all acts of physical, sexual, psychological or economic violence that occur within the family or domestic unit or between former or current spouses or partners, whether or not the perpetrator shares or has shared the same residence with the victim."

This conceptualization acknowledges the complexity of this broad form of aggression, which includes several different typologies of violent conducts, all aimed at attacking, overwhelming and taking advantage of someone who is weak or helpless. ${ }^{2}$ IPV patterns may involve a wide range of physical behaviors, causing injuries to the victim's health and life (e.g. hitting, slapping, shoving, shaking, biting, grabbing, punching, pushing, pinching, strangling, throwing objects, attacking with a weapon or killing), but may also include violence in the form of obstructing medical care, forcing eating or limiting food, as well as acts of sexual violence: that is any act directed at coercing or attempting to coerce any sexual contact or behavior without consent (e.g. sexual assault, sexual harassment, marital rape, unwanted sexual comments). 5,8 Next to these more recognizable and evident kinds of violence acts, others more subtle and difficult to detect also are included as IPV. This includes psychological and emotional coercion aimed at undermining the victim personal self-esteem and personal integrity and dignity (e.g. insulting, humiliating, threatening, offending, intimidating, isolating and controlling behaviors, criticizing, belittling abilities and skills, ignoring), ${ }^{10,11}$ or economic forms of violence, aimed at making the victim financially dependent and increasing the power and control by the abuser (e.g. controlling financial resources, withholding access to money, property and goods, preventing from getting or keeping a job, exclusion from economic decisions). 5 Generally, IPV constitutes a combination of such different forms of violence, interacting with each other; additional manifestations include stalking, to further monitor and control the victim, and aggression or abuse towards children, relatives, property and pets, to harm and intimidate the victim. ${ }^{4}$ While the major- 
ity of IPV is perpetrated by males on female victims, growing recognition and attention is dedicated to male victims and IPV in same sex relationships. ${ }^{6}$

IPV has a variety of significant and potentially devastating short and long-term consequences for victims, affecting their everyday lives, their ability to participate to social life and to express their rights, affecting their health, their social role, disrupting their life and eroding their self-confidence and self-esteem. ${ }^{5}$ It can have negative physical health consequences, immediately and directly connected to the violence, ranging from injuries (e.g. scratches and bruises, lacerations, fractures, weapon wounds) to death, as well as long term lasting health problems, such as worsening of general health conditions (e.g. chronic pain, gastrointestinal disorders, hypertension, gynecological problems and reproductive health issues, HIV/ AIDS and other sexually transmitted diseases, headache, memory loss, reduced mobility, cardiovascular disease). ${ }^{10,12,13}$ Additionally, IPV has notable worsening effects on victims' mental health, leading to the development of psychiatric symptoms and distress (e.g. depression, post-traumatic stress disorder-PTSD, generalized anxiety disorder-GAD, panic disorder-PD, phobias, obsessive-compulsive disorder, somatization, alcohol and substance use disorderSUD, self-harm behaviors). ${ }^{11-14}$ Victims may become even more vulnerable, socially isolated and withdrawn: social isolation and exclusion from the workforce, causing decreased earnings, reduced working ability, or loss of employment, triggering a vicious circle and worsening the victim's situation, as they become increasingly dependent on their abuser. ${ }^{15,16}$

IPV also threatens the stability of families, creating an unhappy, stressful environment characterized by conflict, with negative impacts on individuals, especially children. This also leads to a worsening of social cohesion and may have short- and long-term negative consequences for individual and community safety and health. ${ }^{1,17}$ Moreover, the impact of IPV should be conceptualized not only in terms of the suffering and severely deteriorated quality of life, but also as an economic burden related to increased medical costs (e.g. GP and $\mathrm{A} \& \mathrm{E}$ attendance, hospitalizations, medications), psychosocial care needs (e.g. social services, shelters, therapy), law enforcement efforts (e.g. police, trials, perpetrator treatment, penitentiary system), together with decreased workforce participation and general productivity (e.g. sick leave, job loss, incarceration). ${ }^{18}$

Considering the high toll of IPV on personal and social welfare, the objective of the present paper was to summarize the recent literature on the relationship between IPV and mental health, exploring the risk and vulnerability factors for psychiatric patients becoming victims and/or perpetrators of intimate violent behaviors. We also sought to identify the psychiatric consequences of IPV.

\section{Evidence acquisition}

A review of the recent literature on the topic of IPV and psychiatry was undertaken and reported according to PRISMA statement recommendations. PubMed, Cochrane Library (via OVID) and PsycINFO (via EBSCO) were searched for papers published from November 2011 to November 2016 with the following keywords: ("violence" OR "aggression" OR "homicide" OR "lesion") AND ("domestic" OR "home" OR "partner" OR "intimate partner" OR "wife" OR "husband" OR "child*" OR "parent") AND ("psy*" OR "schiz*" OR "bip*" OR "abuse" OR "substance abuse" OR "trauma" OR "post traumatic stress disorder" OR "PTSD" OR "anx*” OR "depress*” OR "stalking"). Terms were identified by searching titles, abstracts, keywords, medical subject headings and mapping terms to subject headings. The search was restricted to articles using human participants and published in English.

Only papers reporting data from randomized controlled clinical trials, randomized clinical trials, case control studies, cross-sectional studies, longitudinal prospective and retrospective studies were included. Reasons for study exclusion included: absence of clear focus on IPV (off topic); sample sizes smaller than 50 subjects; concerning minors only $(<18$ years of age) or focused on specific sub-pop- 
ulations (ethnic minorities, pregnant women, prisoners, veterans, patients affected by sexually transmitted diseases). Treatment studies on pharmacotherapy or psychotherapy, studies not focused on clinical outcomes (i.e. pharmacological and laboratory research), review articles, books or book chapters, pilot studies or research protocols were similarly not included in this review.

After discarding duplicate records, titles and abstracts were initially screened according to the inclusion and exclusion criteria, and full texts for potentially eligible papers were retrieved. Full-text papers were then screened for their relevance. Data extraction and eligibility assessment were performed independently in an unblinded standardized manner by three pairs of reviewers (G.R. and N.G.; C.S. and L.D.F.; V.M. and A.S.). Disagreements between reviewers were resolved by consensus. The articles thus selected were tabulated detailing: title, first author, year of publication, journal, study design, sample size and population, aim of the study, results, principal conclusions. The table is available on request from the corresponding author.

\section{Evidence synthesis}

The initial bibliographic search yielded 3051 records (1007 from PubMed, 201 from Cochrane Library, and 1843 from PsycINFO), that were reduced to 1486 titles and abstracts to be further screened. In PsychINFO database, only the 278 papers with "Domestic Violence" and "Intimate Partner Violence" as subjects/ major headings were retained. Of these, 897 were subsequently excluded due to being offtopic, 62 because the sample was less than 50 participants, 181 because they concerned minors, 105 because they involved only specific sub-populations. A further 3 were pharmacological studies, 8 were focused on quantitative biological markers and 70 reported outcomes of psychotherapy or medications. A further 50 papers were excluded because they were review articles or Cochrane systematic reviews, and 13 were not included because they were pilot trials or protocols. Therefore, after also

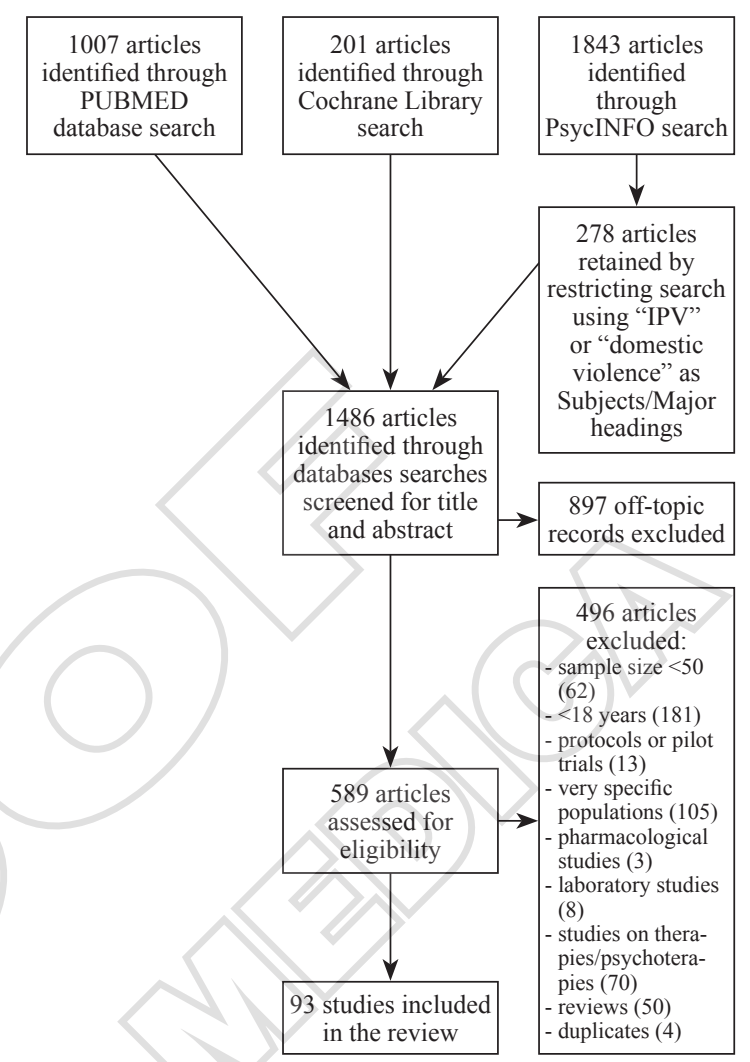

Figure 1.-Flow chart diagram of the selection process of papers for review.

discarding 4 duplicate records, 93 papers were found eligible for inclusion.

Figure 1 shows the selection process followed for this review.

What follows is the narrative summary of findings derived from the included studies. Findings are reported under three main headings: the relationship between mental disorders, substance use disorders and other mental health factors and the risk for perpetrating IPV; the vulnerability of psychiatric patients to become victims of IPV; the mental health consequences of IPV for victims.

\section{Mental health problems and risk for perpetrat- ing IPV}

Researchers have explored the prevalence of mental disorders in perpetrators of IPV in an effort to identify individual risk factors re- 
lated to psychiatric morbidity. The association of IPV with personality disorders, depression and, in particular, PTSD, has been extensively studied and generally confirmed.

A study by Shorey et al. of 88 women arrested for committing domestic violence, found that those reaching diagnostic cut-off scores on self-report screening instruments for mental health problems reported greater IPV perpetration (and also victimization) than women below cut-off scores. Of these, $40.9 \%$ met the cutoff score for depression, $46.6 \%$ for PTSD, $44.3 \%$ for GAD, $35.2 \%$ for panic disorder, $36.4 \%$ for social phobia, $31.8 \%$ for an alcohol use disorder (AUD), and $23.9 \%$ for a SUD. More specifically, bivariate correlations showed that psychological aggression perpetration was significantly associated with symptoms of depression, GAD, PD, and AUD and SUD. Physical aggression and causing physical injury was significantly associated with symptoms of depression, PTSD and GAD. Sexual aggression perpetration was positively related only to PTSD and SUD.19 The same group of researchers found high rates of PTSD and depression in a sample of 308 men arrested for committing IPV, confirming previous research. In addition, the prevalence of GAD, PD, social phobia, and AUD and SUD were very high. Males reaching diagnostic thresholds reported significantly more frequent aggression than males not meeting threshold, even after controlling for social desirability, i.e. the tendency to respond in a socially desirable way. ${ }^{20}$

\section{PTSD}

The psychosocial impact of trauma exposure and PTSD is well recognized. Irritability, poor anger management and aggressiveness are possible PTSD symptoms) ${ }^{21}$ disrupting social functioning and leading to greater family conflict, social isolation, relationship distress, conflicts with intimate partners, and intimate partner aggression.22, 23

Initially only documented in war veterans, more recently the association between IPV perpetration and PTSD has been confirmed in general population samples. For example,
Smith et al., conducted a secondary analysis on a national study of a representative sample of subjects in the USA who reported being in a relationship in the previous year $(25,652$ participants), and found that a PTSD diagnosis in the past year was associated with higher likelihood of reporting perpetration of IPV. Among perpetrators with a PTSD diagnosis in the past year, higher arousal/reactivity symptom cluster scores were associated with greater IPV perpetration for both men and women; intrusion symptom cluster scores were associated with IPV perpetration for men only, whereas negative cognitions/mood symptom cluster scores were only significant among women. ${ }^{24}$

Kirby et al., utilizing both self-report and collateral report of IPV of spouses and partners (in an effort to examine concordance of reports of aggression among cohabitating couples), found that women with PTSD reported more aggressive behaviors in general, and both more IPV perpetration and more victimization. Collateral informants of those with and without PTSD did not differ significantly in their report of IPV by the subject participant. Concordance between participants and spouses or partners was only low to moderate. ${ }^{25}$

In a study exploring the association between maladaptive dependency-related schemas and PTSD hyperarousal symptoms, it was found that high levels of PTSD hyperarousal symptoms, together with greater endorsement of maladaptive dependency schemas, were associated with the perpetration of aggressive behaviors. For those with low levels of hyperarousal symptoms, there was no association between dependency schemas and aggression. ${ }^{26}$

SUD can also influence the interaction between PTSD and IPV. In 288 women with comorbid PTSD-SUD, randomly assigned to a cognitive-behavioral treatment (CBT) focused on trauma and substance abuse symptoms, or to a psychoeducational group, participants who were abstinent at baseline and in the CBT arm were significantly less likely to report IPV over follow-up. Results of the trial suggest that an integrated treatment for PTSD and SUD was associated with significantly better IPV outcomes for a subset of individuals. ${ }^{27}$ 
In path analyses, the strongest correlation emerged between women's perpetration of IPV and women's IPV victimization. In a community sample of 143 substance-using women victims of IPV, and with low socioeconomic status, PTSD re-experiencing and numbing symptom cluster severity were related to women's perpetration of psychological, mild physical, and severe physical IPV; however, these associations appeared to be driven by alcohol misuse. 28

PTSD can be a consequence of early child abuse and neglect. Using an integrated approach, Swopes et al. showed that PTSD mediates the association between adverse childhood experiences and partner aggression, when emotional self-regulation and emotional intelligence were low. ${ }^{29}$

\section{MOOD DISORDERS}

Bipolar Disorder appears to be associated with IPV perpetration. In a study on a subset of defendants previously diagnosed with a bipolar disorder, PTSD, or attention-deficit hyperactivity disorder (ADHD), compared to three matched groups with SUD diagnoses only, individuals diagnosed with bipolar disorder or PTSD, but not ADHD, were more likely to perpetrate partner violence than ADHD and SUD patients. ${ }^{30}$ Depression is a well-known risk factor for physical IPV perpetration, as well as for verbal abuse, increased stress levels, marital dissatisfaction, and anger. ${ }^{31} \mathrm{~A}$ follow-up study from birth to 21 years of 1781 mother/offspring dyads found that women in an intimate partnership showed an association between mental health disorders, including depression, and all three forms of IPV, i.e. psychological (emotional), sexual and physical violence. ${ }^{32}$ Data from Wave II of the USA National Epidemiological Survey on Alcohol and Related Conditions (2004-2005), involving 25,778 eligible participants, who had an intimate partner one year before the survey, showed that depression was associated with IPV perpetration $(\mathrm{OR}=2.00)$ and victimization IPV/perpetration $(\mathrm{OR}=1.74)$, while mania was related to IPV perpetration only $(\mathrm{OR}=2.32) .{ }^{33}$ An asso- ciation of IPV with depressive symptoms was documented also by Bazargan-Hejazi et al. ${ }^{34}$

\section{PERSONALITY DISORDERS}

In a study by Okuda et al., of 1677 individuals who reported perpetrating IPV, IPV perpetrators had greater odds of suffering from any psychiatric disorder compared to non-IPV perpetrators. After adjusting for confounding variables, being younger, having an AUD, a personality disorder, low levels of social support, and low income were associated with perpetration. ${ }^{35}$ In the study among 88 women arrested for committing domestic violence by Shorey et al., ${ }^{19} 29.5 \%$ of the sample met the cutoff score for a probable borderline personality disorder (BPD) diagnosis, and 39.8\% met the cutoff score for a probable antisocial personality disorder (ASPD) diagnosis. For axis II conditions psychological aggression perpetration was positively and significantly correlated with symptoms of BPD and ASPD. Physical aggression and inflicting injury were positively and significantly associated with symptoms of BPD and ASPD. Examining data from a sample of participants ( $46 \%$ male) involved in an intimate relationship one year prior to the survey, Reingle et al. $^{33}$ found an association between ASPD and IPV perpetration $(\mathrm{OR}=2.53)$. According to Rock et al. not only antisocial traits, but other psychopathy traits, should be considered in the prediction of poor treatment response and recidivism in domestic violence offenders. ${ }^{36}$

\section{EMOTIONAL AND PSYCHOLOGICAL TRAITS}

Psychological traits often associated with mental disorders, can influence the risk for committing IPV. Findings from a large national study of the US population showed that impulsivity was related to the risk of dangerous behaviors such as disinhibition, attentional deficits, and lack of planning, including domestic violence. Moreover, self-reported impulsivity was strongly associated with mental disorders (particularly ADHD, SUD and bipolar disorder) and potentially dangerous risk-taking and aggressive behaviors. ${ }^{37}$ 
A recent cross-sectional study on 355 subjects exploring risk factors for offending, found that low self-control predicted men's IPV, whereas anger better predicted women's IPV. ${ }^{38}$

An interactive effect was found between emotion regulation and both PTSD symptoms and negative partner attributions in predicting IPV female-perpetration in a community sample of 254 women, suggesting that women with poor emotion regulation may be at significant risk of perpetrating IPV, regardless of their PTSD symptomatology. 39

Unpopularity, daring, impulsivity, aggressiveness and low verbal IQ in adolescence were individual predictors of adult violent behaviors in intimate relationships, according to a 40-year long prospective longitudinal study on 411 male subjects. 40

Situational factors like self-control depletion and negative emotions (anger, distress, irritation, displeasure) can collectively increase the risk of aggressive behaviors. In a laboratory-based study of 59 heterosexual couples during an in vivo verbal discussion, researchers at the University of Nebraska-Lincoln, in a labbased study among 59 heterosexual couples, found that self-control depletion and emotion reactivity interacted in predicting women's partner aggression, and that negative emotion predicted aggressive behaviors against the partner in men. These results highlight the importance of both individual and dyadic processes in the perpetration of IPV. 41

\section{SUBSTANCE ABUSE/DEPENDENCE}

Another prominent risk factor for IPV perpetration is substance use. ${ }^{31}$ In a recent study by Afifi et al. all types of SUD were associated with increased odds of IPV perpetration. Alcohol and cannabis had the most robust relationship with perpetration of IPV, remaining significant after adjusting for sociodemographic variables, mood disorders, anxiety disorders, personality disorders, and mutual violence. ${ }^{42}$ Cannabis use disorders ${ }^{43}$ and specific combinations of AUD/ SUD (alcohol in combination with cannabis and/or cocaine) appeared to be associated with IPV, particularly in males. ${ }^{44}$ Phencyclidine use may be associated with greater violence perpetration than cannabis used alone or in conjunction with problematic alcohol use..$^{45}$

Data also show that alcohol consumption/ dependence $46-48$ and alcohol use during episodes of IPV is common. ${ }^{44}$ According to Stuart et al., women were more likely to perpetrate physical violence on drinking days relative to non-drinking days. Alcohol use was temporally associated with physical violence perpetration and victimization, and cocaine use was temporally associated with sexual coercion victimization. 49 Finally, studying women in intimate partnerships, Salom et al. showed that AUDs were linked to psychological IPV and illicit SUDs to physical IPV. Moreover, the co-occurrence of related disorders was more strongly linked to psychological and physical IPV. ${ }^{2}$ Interestingly, according to Weiss et al., alcohol use could have a moderating role in the association between IPV and avoidance coping strategies in women. When used in moderation, alcohol consumption may be an adaptive strategy for coping with relational conflict among women who experience IPV.46 In 147 women outpatient victims of IPV, moderate levels of avoidance coping were associated with lower levels of drug use problems, whereas high and low levels of avoidance coping were associated with higher levels of drug use problems. 46

\section{ChILDHOOD EXPOSURE TO DOMESTIC VIOLENCE AND ADULT PERPETRATION OF IPV}

The association between childhood exposure to domestic violence and later IPV perpetration in adulthood has been well-established in the literature. Based on a review of 21 studies, Delsol and Margolin found that from 34\% to $54 \%$ of men who engaged in IPV as adults were exposed to domestic violence as children, compared with the range of $8 \%$ to $27 \%$ of men without a history of violence in their childhood home. ${ }^{50}$ Exposure to domestic violence in childhood can contribute to the normalization and legitimization of violence, and could predict future adult IPV perpetration. ${ }^{51}$ Findings suggest that perpetrators with a family history of violence more strongly endorse 
ideas that present women and feminine attributes in a negative light. ${ }^{52}$ Confounding factors in this association may include socioeconomic disadvantage, family dysfunction, and impaired parental bonding. 51 In particular, a body of evidence indicates that, in children, exposure to IPV toward the mother is one of the most common factors associated with male perpetration and female experience of IPV later in life. ${ }^{53,54}$ With reference to the different types of IPV, in a sample of dissociative disorders outpatients, Webermann et al. found that physical and emotional child abuse were associated with the risk of committing physical IPV, while childhood witnessing of domestic violence and childhood neglect were associated with the risk of perpetration in adulthood of emotional IPV. ${ }^{55}$ Family factors such as having a father with criminal convictions, a disrupted family, poor supervision and relationship problems with parents predicted later IPV. 40 According to Millet et al., IPV perpetration rates were higher among individuals with a history of child maltreatment than in control participants and higher in maltreated men than in women. For men, maltreatment had both direct and mediated effects on IPV perpetration through violent delinquency. ${ }^{56}$ It should also be noted that not only childhood, but also adolescence is a period of risk. In a longitudinal study Sunday and colleagues explored the impact of adolescent physical abuse (occurring between the ages of 12 and 18) in 198 IPV perpetrators. As adults, participants with abuse histories during adolescence showed significantly higher rates of IPV and verbal aggression than did comparison participants: adults with physical abuse histories were more than twice as likely to be physically violent and almost six times more likely to be verbally aggressive to their intimate partners than were comparison participants. ${ }^{57}$

\section{RELATIONSHIP STYLE AND RISK FOR COMMITTING IPV}

Relationship factors associated with the risk of violence perpetration by men include conflict or dissatisfaction in the relationship and male dominance in the family. ${ }^{58}$ Perceiving one's partner as controlling was also found to be significantly associated with physical violence; jealousy and feeling controlled by one's partner were also significant predictors of verbal aggression. ${ }^{57}$ Cohabiting and previous victimization are strongly related to the risk for committing IPV in both genders, according to an American study of 798 men and 770 women who were married or cohabiting. ${ }^{59}$ Exploring the diurnal temporal relationships of environmental, relational and violence variables in violent marital relationships, it was found that previous-day's violence by the husband, lack of husband's alcohol intake, emotional upset and marital distance were significantly related to subsequent husband-perpetrated violence. Wife violence depended upon previous-day violence and alcohol intake only. Moreover, circular causal patterns were noted for marital distance and feeling upset. 60

\section{OTHER RELATED FACTORS}

Recent research suggests interesting contextual effects of social capital on IPV. Higher levels of perceived neighborhood problems, economic disadvantages and low perceived social capital can reflect disadvantaged environments which do not promote health and regulate dysfunctions, and can create stressors which make IPV more likely to occur. ${ }^{61}$ In women, low educational level and discrimination are associated with committing IPV, while younger age, poorer self-perceived health and, interestingly, physical symptoms from irritable bowel syndrome and insomnia are associated with IPV perpetration by men. Understanding these associations may help primary care physicians in identifying male patients who perpetrate IPV. ${ }^{62}$

\section{Risk factors and vulnerability of psychiatric patients to become victims}

IPV is a heterogeneous phenomenon, including the characteristics of IPV-victims, but the topic has been investigated less thoroughly than the characteristics of IPV-perpetrators. 
Most of the literature on IPV victimization concerns the general population, and has revealed a variety of factors that appear to be associated with an increased likelihood of victimization. These include: younger age, ${ }^{61,63-65}$ being female, ${ }^{61,64}$ married, ${ }^{61,63,64}$ and having a lower socioeconomic status. ${ }^{61,63}$

In the general population, significant differences are also observed, with reference to higher total lifetime exposure to traumatic events and to recent exposure to physical or sexual assault. ${ }^{27}$ Moreover, women with high prevalence of past and current IPV victimization are the most likely to report having witnessed parental violence. 66

Recent research specifically addressing the topic of the vulnerability of psychiatric patients to becoming victims of IPV found that mental health disorders may be associated to all forms of IPV victimization, including physical, psychological and combined severe IPV. ${ }^{32}$ In recent years, several studies have specifically examined the correlation between alcohol/drug abuse and being victim of IPV. Overwhelming consensus was found regarding the higher risk of IPV victimization for individuals with alcohol use problems 27, 49, 63-65 or drug abuse, 19, 27, 32, 42, 63,64 with alcohol use being more strongly related to psychological IPV whereas drug abuse was related to physical IPV. Comorbid SUD (including alcohol) and other mental health disorders further increase the likelihood of experiencing IPV. ${ }^{32}$ In particular, cocaine abuse was identified as a risk factor for IPV victimization: $42,64 \mathrm{In}$ 2013, Stuart et al. found that, in comparison to other drugs, days of cocaine use were associated with a six-fold increase in odds of sexual coercion victimization by partner, relative to days of no cocaine use. In contrast, opiate and cannabis use seems to be associated to a lower risk of victimization. ${ }^{49,}, 64$ A common problem of research studies in this area is their substantial difficulty in reliably determining retrospectively whether the violence precedes, coincides with or follows the associated substance misuse.

Women suffering from GAD, panic disorder, bipolar disorder, ASPD and PTSD report more physical, psychological, sexual and injury victimization. ${ }^{19}$ PTSD is not only the major psychiatric risk factor for being an IPV perpetrator, but is also the most common mental health problem reported as a consequence of IPV. 19, 25 Furthermore, PTSD may be associated with IPV revictimization, but there is no agreement on which PTSD symptoms are better predictors of revictimization. Kujipers et al., found that all re-experiencing, arousal, avoidance and numbing symptoms were higher in women who experienced physical IPV revictimization before revictimization, though significantly so only for "re-experiencing" symptoms; 67 Iverson et al. found the same for hyperarousal. 68 It has been hypothesized that women with PTSD, because of higher irritability and anger, may experience greater relationship conflict than women without PTSD, leading to greater IPV both victimization and perpetration. ${ }^{25}$ It has also been suggested that dissociation and disengagement coping styles were associated to IPV re-victimization. ${ }^{68}$

\section{Mental health consequences of IPV}

Several papers have documented the health consequences of IPV. The main negative effects reported are physical injuries and the development of mental distress and disorders including depression, PTSD, sleep difficulties, eating disorders, and suicide attempts. ${ }^{69-71}$ Dissociative symptoms such as subjective feelings of detachment, enhanced perception of imagery, reality distortion, depersonalization, and derealization are also described in IPV survivors. ${ }^{72}$ Depressive symptoms and other psychiatric problems may impair a victim's ability to leave an abusive partner, thereby placing them at risk for continued victimization. ${ }^{14}$ Mental illness has a significant effect, above and beyond sociodemographic variables, in accounting for accessing mental health treatment, where PTSD severity moderated the relationship between perceived need and service use. ${ }^{73}$ Specific psychiatric disorders such as PD and SUD may have also a significant impact on access to services. ${ }^{74}$ 
IPV TYPOLOGIES AND DIFFERENT CONSEQUENCES ON HEALTH

Despite IPV not being a homogenous phenomenon, ${ }^{75}$ all IPV forms have significant impact on victims' short- and long-term psychological well-being of victims, regardless of age. ${ }^{10}$ Data from a 7 -year prospective study demonstrate that higher levels of physical abuse were associated with higher anxiety symptoms, while higher levels of sexual abuse were associated to higher somatization and PTSD symptoms. ${ }^{76}$ As physical and sexual IPV frequently co-occur, women who experience both types of violence are at risk of poorer health outcomes than women who experience only physical violence, particularly of experiencing chronic pain. Higher levels of pain severity and pain interference were significantly associated with anxiety, PTSD, and depressive symptoms. ${ }^{71}$ Also, legal and administrative aggression, defined as the manipulation of legal and administrative resources in an attempt to control or inflict emotional and financial harm on one's partner has been shown to be associated with IPV victims' PTSD and depressive symptoms. ${ }^{77}$

PREVALENCE OF MENTAL DISORDERS IN VICTIMS OF IPV

PTSD, depression and anxiety are, on average, 2 to 4 times more prevalent in IPV victims than in the general population. ${ }^{16,} 78$ Similarly, rates of SUD among women who have experienced IPV are higher than those in the general population. ${ }^{79}$ Sleep disturbances such as insomnia and nightmares were highly prevalent and strongly associated with mental health burden such as PTSD and depression. ${ }^{80}$ Recent surveys suggest that victims of partner violence are also at increased risk of reporting psychotic experiences, characterized by elevated levels of paranoia and auditory hallucinations, ${ }^{81}$ although the evidence supporting this association is still limited. ${ }^{78}$ PTSD and depression often co-occur among women with histories of IPV; some studies highlighted that major depression rarely occurs in the absence of PTSD in this population. ${ }^{75}$ Comorbid mental health conditions (e.g. SUD) can be exacerbated by IPV-related depression and PTSD, which can also delay access to treatment. ${ }^{11}$

Since both genders can be IPV victims, mental health outcomes are worse than in the general population not only in women, but also in men. ${ }^{77}$ According to Hines et al., PTSD and depressive symptoms are the major mental health concerns in male victims, and their severity is related to the severity of the aggression. 82,83

The severity of psychological distress, especially for anxiety and PTSD, increased with the severity and extent of victimization 16 or drug and alcohol problems. ${ }^{66}$ Women who have experienced repeated victimization and/ or multiple types of victimization show a greater prevalence of mental health problems and substance use than women who have experienced a single episode of victimization. 69 Golder et al., identify three groups of women characterized by distinct patterns of current and past victimization: the high IPV/high childhood abuse and neglect (CAN) victimization group reported the highest levels of distress (i.e., IPV-related PTSD symptoms, depression, avoidant coping) and substance use (i.e., greatest number of illicit drugs used; highest percentage of respondents reporting problems associated with substance use). ${ }^{75}$

Different mental disorders were associated with distinct patterns of interpersonal violence and abuse (IVA) (including IPV) in women across their lifespan, suggesting that these IVA patterns correspond to different etiologic pathways. ${ }^{84}$ Behavioral consequences are also described in relation to specific form of IPV. Of the four PTSD symptom clusters, for example, only avoidance symptom severity mediated the relationship between psychological IPV and sexual risky behavior. ${ }^{85}$

\section{PTSD}

PTSD is one of the most prevalent disorders found and studied in IPV victims ${ }^{86}$ with rates estimated to range between $31 \%$ to $84 \% .87,88$ 
In particular, IPV victims compared with people exposed to non-interpersonal trauma were significantly more likely to endorse core symptoms (intrusive reexperiencing, avoidance of reminders, hyperarousal) of PTSD. ${ }^{70} \mathrm{~A}$ prospective study in a sample of 147 residents of battered women's refuges found 6-month follow up rates of $62 \%$ of women experiencing chronic PTSD and 58.2\% met criteria for PTSD or subthreshold PTSD 6 months after leaving a shelter. 89

PTSD is associated with significant physical and mental morbidity. 68,88 It is suggested that PTSD symptoms may mediate the effects of traumatic injury on health outcomes and use of pain killers and psychiatric medications. ${ }^{90}$

Some studies have investigated rates of PTSD symptom presentation and severity after IPV or IPS(sexual)V exposure. ${ }^{87,} 91$ While higher severity of IPV victimization seems to be connected with a higher severity of PTSD symptoms, variability in the response to IPV (in term of nature and extent of trauma-related distress, functional impairment and cooccurring mental health problems) also exists among women who experience bidirectional IPV, i.e. where both partners commit and are victimized by IPV. ${ }^{91}$ In 2015, Hebenstreit et al. described five PTSD symptoms profiles in women's PTSD symptom presentation after IPV exposure: low symptoms ( $46 \%$ of the sample); low symptoms with high hypervigilance $(17 \%)$; intermediate symptoms $(16 \%)$; intermediate symptoms with high hypervigilance $(11 \%)$; and high symptoms $(10 \%) .87$ The most commonly reported PTSD symptom was hyperarousal, followed closely by avoidance. ${ }^{92}$ These findings are consistent with those of Cox et al., among a national sample of US adults $(\mathrm{N} .=2498)$ : in multivariate analysis the variables interpersonal assault, witnessing interpersonal assault and number of reported traumatic events had the strongest association with PTSD D2 and D3 symptoms (D2-persistent negative beliefs and expectations about oneself or the world; D3persistent distorted blame of self or others for the cause or consequences of the traumatic event). ${ }^{21,93}$

\section{OTHER POST-TRAUMATIC SYMPTOMS}

Shame, guilt and self-blame have been recognized for many years as important emotional states in traumatized individuals, moderating the association between different types of abuse and psychological outcomes, including self-esteem and PTSD. ${ }^{94,} 95$ Researching dysfunctional trauma-related cognitions (negative thoughts about the world and self-blame), Beck et al., found that higher levels of shame and depression were significantly associated with higher levels of negative thoughts about the self; higher levels of shame and guilt and lower levels of depression were associated with increased levels of self-blame. An increased level of guilt was the only significant factor associated with negative thoughts about the world 96 .

The literature is inconsistent with regard to the impact of childhood maltreatment on PTSD symptoms among IPV survivors. In a sample of 425 women seeking help for recent victimization, IPV demonstrated significant positive associations with all four PTSD symptom clusters, while childhood maltreatment was positively associated with three of the four PTSD symptom clusters (re-experiencing, numbing and hyperarousal). The authors concluded that IPV did not mediate the association between childhood maltreatment and PTSD symptoms. ${ }^{97}$

\section{DEPRESSION}

IPV results in increased rates of depression, as found by Rollins et al. in 2012 in a sample of 278 women reporting recent IPV: in these women, the mean score on the C-ESD (Center for Epidemiologic Studies Depression Scale, a screening test for depression and depressive disorder) was 31.87 , where a score of 16 or greater is considered indicative of clinical depression. ${ }^{86}$ Research also supports a moderate, longitudinal association between specific forms and severity of IPV/A (physical, sexual, psychological) and major depression or particular patterns of depressive symptoms, although causality cannot be inferred. ${ }^{14}$ Recent 
findings from a population-based longitudinal study of 1052 mothers exposed to partner violence in the UK showed a two- to threefold increased risk of developing new-onset depression and psychosis spectrum symptoms which remained at similar magnitudes over a 2-year period. ${ }^{78}$ Moreover, a longitudinal study among 512 women married or cohabiting with their partner found that depressive symptoms mediate the negative both concurrent and longitudinal association between IPV and employment instability. ${ }^{98}$

\section{Substance, ALCOHOL AND NICOTINE USE}

As already mentioned, IPV is associated with increased substance use 32, 66 alcohol use and alcohol dependence in particular, ${ }^{99}$ possibly as form of attempted self-medication attempts in response to distress caused by victimization. Similarly, a two to three-fold increased prevalence of cigarette smoking was found among women who experience IPV, again possibly as a means of self-medication or as compensatory gratification. ${ }^{100}$ Although all types of SUD are implicated, those that showed the most robust relationship with IPV perpetration and victimization included alcohol and cannabis. ${ }^{42}$ It has been hypothesized that IPV-related PTSD and depressive symptoms mediate the association between IPV and SUD (including alcohol), but research findings are mixed. 88,101

\section{MENTAL HEALTH CONSEQUENCES FOR CHILDREN}

Children of parents experiencing posttraumatic stress symptoms (PTSS) are at increased risk for emotional and behavioral problems. 77,102 Also, the type of abuse experienced by mothers seems to exert different effect on their children: sexual abuse was related to child internalizing problems and higher levels of total psychological problems, whereas physical abuse was related to externalizing problems. ${ }^{76}$ The level of physical and sexual aggression severity endured by the parent also affects the health of children of male IPV victims; only one survey addressed this topic, finding some bivariate associations depending on the child's age: for preschool children, men's reported level of sexual aggression victimization significantly correlated with affective, oppositional defiant, and pervasive development problems, while among the school-age children, father's reported severity of sexual IPV victimization was associated with all of the mental health problems assessed in the children. On a multivariate level, fathers' sexual PV victimization severity was significantly and uniquely associated with two mental health issues in children: attention deficit and affective problems. ${ }^{82}$

\section{HYPOTHESES ON HOW IPV AFFECTS VICTIMS' MENTAL HEALTH}

Several mechanisms can explain the various effects of IPV on the health of the victims. Certain health conditions may directly result from IPV, others may be in relation to maladaptive coping in response to IPV, and yet others may be associated to the biological response to IPV-related stress. ${ }^{82}$ particularly the functioning of the involving for example the hypothalamic-pituitary-adrenal (HPA) axis. HPA axis alterations have been frequently found in PTSD and major depressive disorder. To explain the frequent co-occurrence of PTSD/Major Depressive Disorder (MDD), a common pathway involving HPA dysfunctions has been hypothesized. A study examining the relation of comorbid PTSD/MDD to HPA circadian dynamics associated with IPV concluded that women with comorbid PTSD and MDD showed the largest cortisol waking response and that comorbid MDD drove the elevated cortisol waking response originally observed in PTSD. ${ }^{103}$ Other findings suggest that low awakening cortisol levels may be associated with a dissociative coping style developed in the context of IPV trauma exposure. ${ }^{72}$

Exposure to IPV trauma can impact negatively on fundamental areas of intrapersonal functioning such as attachment processes, emotion regulation and cognitive assumptions. The impairment of these areas seems to be linked to an increased risk for post-traumatic psychopathology. In a study of female healthcare workers experiencing IPV the association 
between victimization and depressive symptoms was found to vary according to the level of anxious attachment: specifically, women with higher anxious attachment insecurity were more vulnerable to develop depressive symptoms. In contrast to previous research, neither avoidant nor anxious attachment moderated the association between IPV and PTSD symptoms. ${ }^{11}$ In two different samples of survivors of interpersonal trauma, emotion dysregulation was the most consistent predictor of mental health problems, followed by negative cognitions regarding the world, self, and others. ${ }^{104}$ Also, women with violent partners developed a perception of themselves as damaged, leading to negative self-worth, low self-esteem, poor self-image, or decreased assertiveness and self-confidence, higher than in the comparison group. ${ }^{105}$

Interpersonal style may influence in different ways the degree to which women exhibit IPV-related psychological problems. Examining the two interpersonal style dimensions of dominance and warmth, Yalch and Levendosky have recently found that both were associated with less negative (i.e., less betrayed, self-blaming, fearful, alienated, angry, and shameful) appraisals of IPV over and above the effect of IPV severity. ${ }^{106}$ Effects were generally strongest for dominance, exhibiting smallto medium-sized negative effects, confirming previous research that identified dominance as a protective factor against the effects of IPV. Perceived relationship power was significantly and negatively associated with mental health issues: among substance-using incarcerated women, less perceived relationship power was associated with more mental health issues. Perceived relationship power was significantly and negatively associated with the number of IPV experiences. 107

In order to understand whether coping strategies change over the course of a violent relationship, recent research has addressed women's IPV-related coping strategies (such as problem-focused coping and emotion-focused coping). Lokhmatkina et al. and Sullivan et al. observed that use of active forms of coping, such as problem solving or social support cop- ing, is associated with decreased risk for alcohol use, whereas avoidant coping strategies has been traditionally associated with negative long-term effects (maintenance of traumainduced symptoms and poorer mental health outcomes). 100, 108 Avoidance coping mediated the relationships between psychological and sexual IPV victimization and the outcomes of PTSD symptom severity, depression severity, drug use problems, whereas it did not influence the effects of physical IPV victimization on mental issues. ${ }^{109}$ However, flexible, situationally-appropriate use of avoidance coping may be an adaptive strategy in drug use problems in IPV-exposed women. Moderate levels of avoidance coping were associated with lower levels of drug use problems, whereas high and low levels of avoidance coping predicted higher levels of drug use problems. ${ }^{46}$

Social problem solving is also impaired by post-IPV trauma: a predominately negative problem-solving approach was strongly associated with poorer outcomes (having clinically significant levels of PTSD, anxiety, depression, and somatization) for both mothers and children in the aftermath of abuse. ${ }^{110}$ Consistent with more general avoidance coping, avoidant problem solving served as an intermediating variable for the relationship between abuse exposure and PTSD severity. ${ }^{111}$

Social factors are often central to risk and recovery from mental disorders in the aftermath of interpersonal trauma. There is evidence that receiving higher social and emotional support (e.g. professional - individual and group therapy - or from family and/or friends) significantly reduces risk for poor mental and physical health, anxiety, current depression, PTSD symptoms, and suicide attempts. ${ }^{69,}, 89,112$ In order to test the psychological impact of institutional support in the relationship with IPV survivors' psychological outcomes, Srinivas and DePrince looked at the police response as a primary and integral part of support. They found that a more negative police response, or unmet women's expectations in relation to the police, was significantly associated with greater PTSD symptom severity. ${ }^{113}$

Few studies have examined attachment 
style and social support together in the context of psychopathology: in a sample of 108 IPV victims, higher anxious attachment style and lower social support were found significantly associated with higher self-reported PTSD and higher self-reported depression, although only lower social support was related to clinician assessed PTSD and depression. Only higher attachment anxiety was associated with self-reported generalized anxiety disorder, whereas lower attachment dependency showed a significant association to clinician assessed GAD. 114

Empowerment and John Henryism (a coping strategy consisting in exerting high levels of effort in dealing with social stresses, resulting in the accumulation of physiological consequences) as a resilience factor were demonstrated to have protective effects attenuating the impact of IPV severity on PTSD at low and moderate levels of violence 115 and on depressive symptoms although with some differences between black and white women. ${ }^{116}$

Table I summarizes mental health risk factors for perpetrating IPV, vulnerability factors for becoming victims and mental health consequences emerging from the reviewed papers.

TABLE I.-Key mental health factors connected to interpersonal partner violence (IPV) emerging from published studies 2011-2016.

Mental health risk factors for committing IPV

- Alcohol and substance use disorders

- PTSD, bipolar disorder, depression, generalized anxiety disorder

- Borderline personality disorder, antisocial personality disorder

- Impulsivity, low self-control, anger, jealousy

- Exposure to IPV during childhood and adolescence

Mental health risk factors for becoming victims of IPV

- Alcohol and substance use disorders

- PTSD, generalized anxiety disorder, panic disorder

- Borderline personality disorder, antisocial personality disorder

Mental health consequences of IPV

- Depression, PTSD, sleep difficulties, eating disorders, suicide attempts

- Anxiety, somatization

- Chronic pain

- Alcohol and substance use disorders

PTSD: post-traumatic stress disorder.

\section{Conclusions}

IPV is, unfortunately, a very common phenomenon with severe negative mental health consequences. Moreover, various mental disorders increase the risk of becoming a perpetrator and/or a victim of IPV, thus creating a vicious cycle. Nevertheless, IPV appears to be an underestimated problem in the day-to-day running of many mental health services. Selfdisclosure about experiences of IPV can be difficult both for patients, who may be afraid of the potential consequences of such a disclosure, and for mental health professionals, who often complain about their lack of knowledge and expertise in this area and may even question if IPV concerns are part of their job.117

Mental health professionals should contribute to overcoming barriers to disclosure and should explore IPV experiences in the life of their patients. The diagnostic evaluation and assessment of IPV perpetrators and victims could provide the opportunity to identify and treat psychiatric disorders and improve the recovery from conditions that can increase the risk of committing or suffer further violence. 35 Specific training in the early detection of IPV could help mental health workers to provide support to victims, offer treatment to perpetrators and facilitate collaboration with social services and child and adolescent mental health services.

Primary and secondary prevention programs should focus on factors that mediate the association between childhood exposure to IPV and perpetration of IPV in adulthood..$^{51}$

Finally, further research efforts are needed to elucidate the risk factors for victimization, and to develop systemic and prevention-oriented responses.

\section{References}

1. World Health Organization, London School of Hygiene and Tropical Medicine. Preventing intimate partner and sexual violence against women. Taking action and generating evidence. Geneva: WHO Press; 2010.

2. World Health Organization. Global and regional estimates of violence against women: prevalence and health effects of intimate partner violence and non-partner sexual violence. Geneva: WHO Press; 2013. 
3. Giolo O. Le "periferie" del patriarcato. L'uguaglianza, i diritti umani e le donne. In: Casadei T, editor. Diritti umani e soggetti vulnerabili. Torino: Giappichelli; 2012. p. $119-42$.

4. Sgarbi C. La violenza contro le donne e il fenomeno dello stalking. In: Rescigno F, editor. Percorsi di Eguaglianza. Torino: Giappichelli; 2016. p. 133-53.

5. United Nations. The World's Women 2015: Trends and Statistics. New York: United Nations, Department of Economic and Social Affairs, Statistics Division; 2015.

6. 6 Smith SG, Chen J, Basile KC, Gilbert LK, Merrick MT, Patel N, et al. The National Intimate Partner and Sexual Violence Survey (NISVS): 2010-2012 State Report. Atlanta, GA: National Center for Injury Prevention and Control, Centers for Disease Control and Prevention; 2017.

7. European Institute for Gender Equality. Review of the Implementation of the Beijing Platform for Action in the EU Member States: Violence against Women - Victim Support. Luxembourg: Publications Office of the European Union; 2012.

8. European Union Agency for Fundamental Rights. Violence Against Women: An EU wide-survey. Luxembourg: Publications Office of the European Union; 2014.

9. Council of Europe. Convention on preventing and combating violence against women and domestic violence; 2011 [Internet]. Available from: www.conventions.coe. int/Treaty/ITA/Treaties/Html/210.htm [cited 2017, Jul 17].

10. Stöckl H, Penhale B. Intimate Partner Violence and Its Association With Physical and Mental Health Symptoms Among Older Women in Germany. J Interpers Violence 2015;30:3089-111.

11. La Flair LN, Bradshaw CP, Mendelson T, Campbell J. Intimate Partner Violence and Risk of Psychiatric Symptoms: the Moderating Role of Attachment. J Fam Violence 2015;30:567-77.

12. Wong J, Mellor D. Intimate partner violence and women's health and wellbeing: impacts, risk factors and responses. Contemp Nurse 2014;46:170-9.

13. Howard LM. Domestic violence: its relevance to psychiatry. Adv Psychiatr Treat 2012;18:129-36.

14. La Flair LN, Bradshaw CP, Campbell J. Intimate Partner Violence/abuse and Depressive Symptoms among Female Health Care Workers: Longitudinal Findings. Women's Health Issues 2012;22:e53-9.

15. Galano MM, Hunter EC, Howell KH, Miller LE, Graham-Bermann SA. Predicting Shelter Residence in Women Experiencing Recent Intimate Partner Violence. Violence Against Women 2013;19:518-35.

16. Ferrari G, Agnew-Davies R, Bailey J, Howard L, Howarth E, Peters TJ, et al. Domestic Violence and Mental Health: A Cross-Sectional Survey of Women Seeking Help from Domestic Violence Support Services. Global Health Action 2016;9:29890.

17. Garcia-Moreno C, Watts C. Violence against women: an urgent public health priority. Bulletin of the World Health Organization 2011;89:1-2

18. European Parliament. Policy Department C. Citizens' rights and Constitutional Affairs. The Issue of violence against women in the European Union. Brussels: European Parliament; 2016.

19. Shorey RC, Elmquist J, Ninnemann A, Brasfield H, Febres J, Rothman EF et al. The Association between Intimate Partner Violence Perpetration, Victimization, and Mental Health among Women Arrested for Domestic Violence. Partner Abuse 2012;3:3-21.

20. Shorey RC, Febres J, Brasfield H, Stuart GL. The Prevalence of Mental Health Problems in Men Arrested for Domestic Violence. J Fam Violence 2012;27:741-48.

21. American Psychiatric Association. Diagnostic and
Statistical Manual of Mental Disorders. Fifth Edition. Washington, D.C: American Psychiatric Association; 2014

22. Beckham JC, Roodman AA, Barefoot JC, Haney TL, Helms MJ, Fairbank JA, et al. Interpersonal and selfreported hostility among combat veterans with and without posttraumatic stress disorder. J Trauma Stress 1996;9:335-43.

23. Chrysos ES, Taft CT, King LA, King DV. Gender, partner violence, and perceived family functioning among a sample of Vietnam veterans. Violence Vict 2005;20:54959.

24. Smith KZ, Smith PH, Violanti, JM, Bartone PT, Homish GG. Posttraumatic Stress Disorder Symptom Clusters and Perpetration of Intimate Partner Violence: Findings From a U.S. Nationally Representative Sample. J Trauma Stress 2015;28:469-74.

25. Kirby AC, Beckham JC, Calhoun PS, Roberts ST, Taft $\mathrm{CT}$, Elbogen EB, et al. An Examination of General Aggression and Intimate Partner Violence in Women with Posttraumatic Stress Disorder. Violence Vict 2012;27:777-92.

26. Kachadourian LK, Taft CT, Holowka DW, Woodward H, Marx BP, Burns A. Maladaptive Dependency Schemas, Posttraumatic Stress Hyperarousal Symptoms, and Intimate Partner Aggression Perpetration. J Trauma Stress 2013;26:580-7.

27. Cohen LR, Field C, Campbell ANC, Hien DA. Intimate Partner Violence Outcomes in Women with PTSD and Substance Use: A Secondary Analysis of NIDA Clinical Trials Network 'Women and Trauma' Multi-Site Study. Addict Behav 2013;38:2325-32.

28. Hellmuth JC, Jaquier V, Young-Wolff K, Sullivan TP. Posttraumatic Stress Disorder Symptom Clusters, Alcohol Misuse, and Women's Use of Intimate Partner Violence. J Trauma Stress 2013;26:451-58.

29. Swopes RM, Simonet DV, Jaffe AE, Tett RP, Davis JL. Adverse Childhood Experiences, Posttraumatic Stress Disorder Symptoms, and Emotional Intelligence in Partner Aggression. Violence Vict 2013;28:513-30.

30. Crane CA, Hawes SW, Devine S, Easton CJ. Psychopathology and the Perpetration of Intimate Partner Violence. J Clin Psychol 2014;70:238-47.

31. Schumacher JA, Feldbau-Kohn S, Smith Slep AMS, Heyman RE. Risk factors for male-to-female partner physical abuse. Aggress Violent Behav 2001;6:281-352.

32. Salom CL, Williams GL, Najman JM, Alati R. Substance Use and Mental Health Disorders Are Linked to Different Forms of Intimate Partner Violence Victimisation. Drug Alcohol Depend 2015;151:121-7.

33. Reingle JM, Jennings WG, Connell NM, Businelle MS, Chartier K. On the Pervasiveness of Event-Specific Alcohol Use, General Substance Use, and Mental Health Problems as Risk Factors for Intimate Partner Violence. J Interpers Violence 2014;29:2951-70.

34. Bazargan-Hejazi S, Kim E, Lin J, Ahmadi A, Khamesi MT, Teruya S. Risk Factors Associated with Different Types of Intimate Partner Violence (IPV): An Emergency Department Study. J Emerg Med 2014;47:710-20.

35. Okuda M, Olfson M, Wang S, Rubio JM, Xu Y, Blanco C. Correlates of Intimate Partner Violence Perpetration Results from a National Epidemiologic Survey. J Trauma Stress 2015;28:49-56.

36. Rock RC, Sellbom M, Ben-Porath Y. Concurrent and Predictive Validity of Psychopathy in a Batterers' Intervention Sample. Law Hum Behav2013;37:145-54.

37. Chamorro J, Bernardi S, Potenza MN, Grant JE, Marsh $\mathrm{R}$, Wang $\mathrm{S}$, et al. Impulsivity in the General Population: A National Study. J Psychiatr Res 2012;46:994-1001.

38. Thornton AJV, Graham-Kevan N, Archer J. Intimate Partner Violence: Are the Risk Factors Similar for Men 
and Women, and Similar to Other Types of Offending? Aggress Behav 2016;42:404-12.

39. Price RK, Bell KM, Lilly M. The Interactive Effects of PTSD, Emotion Regulation, and Anger Management Strategies on Female-Perpetrated IPV. Violence Vict 2014;29:907-26.

40. Theobald D, Farrington DP. Child and Adolescent Predictors of Male Intimate Partner Violence. J Child Psychol Psychiatry 2012;53:1242-9.

41. Watkins L, Di Lillo D, Hoffman L, Templin J. Do selfcontrol depletion and negative emotion contribute to intimate partner aggression? University of Nebraska Faculty Publications, Department of Psychology; 2013 [Internet]. Available from: http://digitalcommons.unl. edu/psychfacpub/633 [cited 2017, Jul 17].

42. Afifi TO, Henriksen CA, Asmundson GLG, Sareen J. Victimization and Perpetration of Intimate Partner Violence and Substance Use Disorders in a Nationally Representative Sample. J Nerv Ment Dis 2012;200:684-91.

43. Gonzalez, Reingle JM, Connell NM, Businelle MS, Wesley G. Jennings, et al. Characteristics of Adults Involved in Alcohol-Related Intimate Partner Violence: Results from a Nationally Representative Sample. BMC Public Health 2014;14:466.

44. Kraanen FL, Vedel E, Scholing A, Emmelkamp PMG. Prediction of Intimate Partner Violence by Type of Substance Use Disorder. J Subst Abuse Treat 2014;46:53239.

45. Crane CA, Easton CJ, Devine S. The Association between Phencyclidine Use and Partner Violence: An Initial Examination. J Addict Dis 2013;32:150-7.

46. Weiss NH, Duke AA, Sullivan TP. Evidence for a Curvilinear Dose-Response Relationship between Avoidance Coping and Drug Use Problems among Women Who Experience Intimate Partner Violence. Anxiety Stress Coping 2014;27:722-32.

47. Colorado Yoar SM, Agudelo-Suarez AA, Huerta JM, Torres-Cantero JM. Intimate Partner Violence and Its Associated Factors in a Sample of Colombian Immigrant Population in Spain. J Immigr Minor Health 2016;18:904-12.

48. Stanley S. Intimate Partner Violence and Domestic Violence Myths: A Comparison of Women With and Without Alcoholic Husbands (A Study from India). J Comp Fam Stud 2012;43:647-72.

49. Stuart GL, Moore TM, Elkins SR, O'Farrell TJ, Temple JR, Ramsey SE, et al. The Temporal Association between Substance Use and Intimate Partner Violence among Women Arrested for Domestic Violence. J Consult Clin Psychol 2013;4:681-90.

50. Delsol C, Margolin G. The role of family-of-origin violence in men's marital violence perpetration. Clin Psychol Review 2004;24:90-122.

51. Mbilinyi LF, Logan-Greene PB, Neighbors C, Walker DD, Roffman RA, Zegree J. Childhood Domestic Violence Exposure among a Community Sample of Adult Perpetrators: What Mediates the Connection? J Aggress Maltreat Trauma 2012;21:71-87.

52. Lee RD, Walters ML, Hall JE, Basile KC. Behavioral and Attitudinal Factors Differentiating Male Intimate Partner Violence Perpetrators With and Without a History of Childhood Family Violence. J Fam Violence 2013;28:85-94.

53. Kishor S, Johnson K. Profiling domestic violence - a multi-country study. Calverton, MD: ORC Macro; 2004.

54. Abramsky T, Watts CH, Garcia-Moreno C, Devries K, Kiss L, Ellsberg M, et al. What factors are associated with recent intimate partner violence? Findings from the WHO multi-country study on women's health and domestic violence. BMC Public Health 2011;11:109.

55. Webermann AR, Brand BL, Chasson GS. Childhood
Maltreatment and Intimate Partner Violence in Dissociative Disorder Patients. Eur J Psychotraumatol 2014;5:24568

56. Millett LS, Kohl PL, Jonson-Reid M, Petra M. Child Maltreatment Victimization and Subsequent Perpetration of Young Adult Intimate Partner Violence: An Exploration of Mediating Factors. Child Maltreat 2013:18:71-84.

57. Sunday S, Kline M, Labruna V, Pelcovitz D, Salzinger S, Kaplan S. The Role of Adolescent Physical Abuse in Adult Intimate Partner Violence. J Interpers Violence 2011;26:3773-89.

58. Garcia-Moreno C, Jansen H AFM, Watts C, Ellsberg M, Heise L, et al. WHO multi-country study on women's health and domestic violence against women: initial results on prevalence, health outcomes and women's responses. Geneva: WHO Press; 2005.

59. O'Leary KD, Tintle NL, Bromet E. Risk factors for physical violence against partners in the U.S, 2014. Psychol Violence 2014;4:65-77.

60. Katerndahl D, Burge S, Ferrer R, Becho J, Wood R. Webs of causation in violent relationships. J Eval Clin Pract 2014;20:703-10.

61. Kirst M, Lazgare LP, Zhang YJ, O'Campo P. The Effects of Social Capital and Neighborhood Characteristics on Intimate Partner Violence: A Consideration of Social Resources and Risks. Am J Community Psychol 2015;55:314-25

62. Singh V, Tolman R, Walton M, Chermack S, Cunningham R. Characteristics of Men Who Perpetrate Intimate Partner Violence. J Am Board Fam Med 2014;5:661-8.

63. Regueira-Diéguez A, Pérez-Rivas N, Muñoz-Barús JI, Vázquez-Portomeñe F, Rodríguez-Calvo MS. Intimate Partner Violence Against Women in Spain: a Medicolegal and Criminological Study. J Forensic Leg Med 2015;34:119-26.

64. Chermack ST, Murray R, Kraus S, Walton MA, Cunningham RM, Barry KL, et al. Characteristics and Treatment Interests among Individuals with Substance Use Disorders and a History of Past Six-month Violence: Findings from an Emergency Department Study. Addict Behav 2014;39:265-72.

65. Kramer TL, Borders TF, Tripathi S, Lynch C, Leukefeld C, Falck RS, et al. Physical Victimization of Rural Methamphetamine and Cocaine Users. Violence Vict 2012;27:109-24.

66. Young-Wolff KC, Hellmuth J, Jaquier V, Swan SC, Connell C, Sullivan TP. Patterns of Resource Utilization and Mental Health Symptoms among Women Exposed to Multiple Types of Victimization: A Latent Class Analysis. J Interpers Violence 2013;28:3059-83.

67. Kuijpers KF, Van der Knaap LM, Winkel FW. PTSD Symptoms as Risk Factors for Intimate Partner Violence Revictimization and the Mediating Role of Victims' Violent Behavior. J Trauma Stress 2012;25:179-86.

68. Iverson KM, Litwack SD, Pineles SL, Suvak MK, Vaughn RA, Resick PA. Predictors of Intimate Partner Violence Revictimization: The Relative Impact of Distinct PTSD Symptoms, Dissociation, and Coping Strategies. J Trauma Stress 2013;26:102-10.

69. Svavarsdóttir EK, Orlygsdottir B, Gudmundsdottir B. Reaching Out To Women Who Are Victims of Intimate Partner Violence. Perspect Psychiatr C 2015;51:190201.

70. Forbes D, Lockwood E, Phelps A, Wade D, Creamer M, Bryant RA, McFarlane A, et al. Trauma at the Hands of Another: Distinguishing PTSD Patterns Following Intimate and Nonintimate Interpersonal and Noninterpersonal Trauma in a Nationally Representative Sample. J Clin Psychiatry 2014;75:147-53.

71. Symes L, McFarlane J, Nava A, Gilroy H, Maddoux 
J. The Association of Pain Severity and Pain Interference Levels with Abuse Experiences and Mental Health Symptoms among 300 Mothers: Baseline Data Analysis for a 7-Year Prospective Study. Issues Ment Health Nurs 2013;34:2-16.

72. Basu A, Levendosky AA, Lonstein JS. Trauma Sequelae and Cortisol Levels in Women Exposed to Intimate Partner Violence. Psychodyn Psychiatry 2013;41:247-75.

73. Klopper JJ, Schweinle W, Ractliffe KC, Elhai JD. Predictors of Mental Healthcare Use among Domestic Violence Survivors in Shelters. Psychol Serv 2014;11:13440.

74. Cheng TC. Intimate Partner Violence and Welfare Participation: A Longitudinal Causal Analysis. J Interpers Violence 2013;28:808-30

75. Golder S, Connell CM, Sullivan TP. Psychological Distress and Substance Use among Community-Recruited Women Currently Victimized by Intimate Partners: a Latent Class Analysis and Examination of Between-Class Differences. Violence Against Wom 2012;18:934-57.

76. Symes L, Maddoux J, McFarlane J, Nava A, Gilroy H. Physical and Sexual Intimate Partner Violence, Women's Health and Children's Behavioural Functioning: Entry Analysis of a Seven-Year Prospective Study. J Clin Nurs 2014;23:2909-18.

77. Berger JL, Douglas EM, Hines DA. The Mental Health of Male Victims and Their Children Affected by Legal and Administrative Partner Aggression. Aggress Behav 2016;42:346-61

78. Ouellet-Morin I, Fisher HL, York-Smith M, FinchamCampbell S, Moffitt TE, Arseneault L. Intimate Partner Violence and New-Onset Depression: a Longitudinal Study of Women's Childhood and Adult Histories of Abuse. Depress Anxiety 2015;32:316-24.

79. Weiss NH, Duke AA, Sullivan TP. Probable Post Traumatic Stress Disorder and Women's Use of Aggression in Intimate Relationships: The Moderating Role of Alcohol Dependence. J Trauma Stress 2014;27:550-7.

80. Pigeon WR, Cerulli C, Richards H, He H, Perlis M, Caine E. Sleep Disturbances and Their Association with Mental Health among Women Exposed to Intimate Partner Violence. J Womens Health 2011;20:1923-9.

81. Shevlin M, O'Neill T, Houston JE, Read J, Bentall RP, Murphy J. Patterns of Lifetime Female Victimisation and Psychotic Experiences: a Study Based on the UK Adult Psychiatric Morbidity Survey 2007. Soc Psychiatry Psychiatr Epidemiol 2013;48:15-24.

82. Hines DA, Douglas EM. Sexual Aggression Experiences Among Male Victims of Physical Partner Violence: Prevalence, Severity, and Health Correlates for Male Victims and Their Children. Arch Sex Behav 2016;45:1133-51

83. Hines DA, Douglas EM. Health Problems of Partner Violence Victims: Comparing Help-Seeking Men to a Population-Based Sample. Am J Prev Med 2015;48:136-44.

84. Cavanaugh CE, Martins SS, Petras H, Campbell JC. Mental Disorders Associated with Subpopulations of Women Affected by Violence and Abuse. J Trauma Stress 2013;26:459-66.

85. Overstreet NM, Willie TC, Hellmuth JC, Sullivan TP. Psychological Intimate Partner Violence and Sexual Risk Behavior: Examining the Role of Distinct Posttraumatic Stress Disorder Symptoms in the Partner Violence-Sexual Risk Link. Women's Health Issues: Official Publication of the Jacobs Institute of Women's Health 2015;25:73-8.

86. Rollins C, Glass NE, Perrin NA, Billhardt KA, Clough A, Barnes J, et al. Housing Instability Is as Strong a Predictor of Poor Health Outcomes as Level of Danger in an Abusive Relationship: Findings from the SHARE Study. J Interpers Violence 2012;27:623-43.
87. Hebenstreit CL, Maguen S, Koo KH, DePrince AP. Latent Profiles of PTSD Symptoms in Women Exposed to Intimate Partner Violence. J Affect Disord 2015;180:122-8.

88. Peters EN, Khondkaryan E, Sullivan TP. Associations between Expectancies of Alcohol and Drug Use, Severity of Partner Violence, and Posttraumatic Stress among Women. J Interpers Violence 2012;27:2108-27.

89. Johnson DM, Zlotnick C. Remission of PTSD after Victims of Intimate Partner Violence Leave a Shelter. J Trauma Stress 2012;25:203-6.

90. Cody MW, Beck JG. Physical Injury, PTSD Symptoms, and Medication Use: Examination in Two Trauma Types. J Trauma Stress 2014;27:74-81.

91. Hellmuth JC, Jaquier V, Swan SC, Sullivan TP. Elucidating Posttraumatic Stress Symptom Profiles and Their Correlates among Women Experiencing Bidirectional Intimate Partner Violence. J Clin Psychol 2014;70:1008-21

92. Messing JT, Thaller J, Bagwell M. Factors Related to Sexual Abuse and Forced Sex in a Sample of Women Experiencing Police-Involved Intimate Partner Violence. Health Soc Work 2014;39:181-91.

93. Cox KS, Resnick HS, Kilpatrick DJ. Prevalence and Correlates of Posttrauma Distorted Beliefs: Evaluating DSM-5 PTSD Expanded Cognitive Symptoms in a National Sample. J Trauma Stress 2014;27:299-306.

94. Reich CM, Jones JM, Woodward MJ, Blackwell N, Lindsey LD, Beck JG. Does Self-Blame Moderate Psychological Adjustment Following Intimate Partner Violence? J Interpers Violence 2015;30:1493-510.

95. Beck JG, McNiff J, Clapp JD, Olsen SA, Avery ML, Hagewood JH. Exploring Negative Emotion in Women Experiencing Intimate Partner Violence: Shame, Guilt, and PTSD. Behav Ther 2011;42:740-50.

96. Beck JG, Reich CM, Woodward MJ, Olsen SA, Jones JM, Patton SC. How Do Negative Emotions Relate to Dysfunctional Posttrauma Cognitions? An Examination of Interpersonal Trauma Survivors. PsycholTrauma 2015:7:3-10

97. Gobin RL, Iverson KM, Mitchell K, Vaughn R, Resick PA. The Impact of Childhood Maltreatment on PTSD Symptoms among Female Survivors of Intimate Partner Violence. Violence Vict 2013;28:984-99.

98. Crowne SS, Juon HS, Ensminger M, Burrel L. Concurrent and long-term impact of intimate partner violence on employment stability. J Interpersonal Violence 2011;26:1282-304

99. Golding JM. Intimate partner violence as a risk factor for mental disorders: a meta-analysis. J Fam Violence 1999;14:99-132.

100. Sullivan TP, Flanagan JC, Dudley DN, Holt LJ, Mazure $\mathrm{CM}, \mathrm{McKee}$ SA. Correlates of Smoking Status among Women Experiencing Intimate Partner Violence: Substance Use, Posttraumatic Stress, and Coping. Am J Addict 2015:24:546-53.

101. Sullivan TP, Ashare RL, Jaquier V, Tennen H. Risk Factors for Alcohol-Related Problems among Victims of Partner Violence. Subst Use Misuse 2012;47:673-85.

102. Katz LF, Gurtovenko K. Posttraumatic Stress and Emotion Regulation in Survivors of Intimate Partner Violence. J Fam Psychol 2015;29:528-36

103. Pinna KLM, Johnson DM, Delahanty DL. PTSD, Comorbid Depression, and the Cortisol Waking Response in Victims of Intimate Partner Violence: Preliminary Evidence. Anxiety Stress Coping 2014;27:253-69.

104. Lilly MM, Lim BHP. Shared Pathogeneses of Posttrauma Pathologies: Attachment, Emotion Regulation, and Cognitions. J Clin Psychol 2013;69:737-48.

105. Lynch SM. Not Good Enough and on a Tether: Exploring How Violent Relationships Impact Women's Sense of Self. Psychodyn Psychiatry 2013;41:219-46. 
106. Yalch MM, Levendosky AA. The Influence of Interpersonal Style on the Appraisal of Intimate Partner Violence. J Interpers Violence 2016;31:2430-44.

107. Minieri AM, Staton-Tindall M, Leukefeld C, Clarke JG, Surratt HL, Frisman LK. Relationship Power as a Mediator of Intimate Partner Violence and Mental Health Issues among Incarcerated, Substance-Using Women. Int J Offender Ther Comp Criminol 2014;58:303-19.

108. Lokhmatkina NV, Agnew-Davies R, Costelloe C, Kuznetsova OY, Nikolskaya IM, Feder GS. Intimate Partner Violence and Ways of Coping with Stress: Cross-Sectional Survey of Female Patients in Russian General Practice. Fam Pract 2015;32:141-6.

109. Hellmuth JC, Jaquier V, Overstreet N, Swan SC, Sullivan TP. The mediating role of avoidance coping between ipv victimization, mental health, and substance abuse among women experiencing bidirectional IPV. Psychiatry Res 2014;15:391-6.

110. Maddoux J, Symes L, McFarlane J, Koci A, Gilroy H, Fredland N. Problem-Solving and Mental Health Outcomes of Women and Children in the Wake of Intimate Partner Violence. J Environ Public Health 2014:708;198.

111. Reich CM, Blackwell N, Simmons CA, Beck JG. Social Problem Solving Strategies and Posttraumatic Stress Disorder in the Aftermath of Intimate Partner Violence. J Anxiety Disord 2015;32:31-7.
112. Woodward MJ, Eddinger J, Henschel AV, Dodson TS, Tran HN, Beck JG. Social Support, Posttraumatic Cognitions, and PTSD: The Influence of Family, Friends, and a Close Other in an Interpersonal and Non-Interpersonal Trauma Group. J Anxiety Disord 2015;35:60-7.

113. Srinivas T, DePrince AP. Links between the Police Response and Women's Psychological Outcomes Following Intimate Partner Violence. Violence Vict 2015;30:3248.

114. Woodward MJ, Patton SC, Olsen SA, Jones JM, Reich CM, Blackwell N, Beck JG. How Do Attachment Style and Social Support Contribute to Women's Psychopathology Following Intimate Partner Violence? Examining Clinician Ratings versus Self-Report. J Anxiety Disord 2013;27:312-20.

115. Perez S, Johnson DM, Wright CV. The Attenuating Effect of Empowerment on IPV-Related PTSD Symptoms in Battered Women Living in Domestic Violence Shelters. Violence Against Women 2012;18:102-17.

116. Kramer NM, Johnson NL, Johnson DM. Is John Henryism a resilience factor in women experiencing intimate partner violence? Anxiety Stress Coping 2015;28:60116.

117. Hegarty K. Domestic violence: the hidden epidemic associated with mental illness. Br J Psychiatry 2011;198:169-70

Conflicts of interest.-The authors certify that there is no conflict of interest with any financial organization regarding the material discussed in the manuscript.

Acknowledgements.-The authors wish to thank Kathryn Elkins and Andrew Mackinnon (Melbourne, VIC, Australia) for offering invaluable help in revising form and language of earlier drafts of this review.

Manuscript accepted: June 20, 2017. - Manuscript received: June 14, 2017.

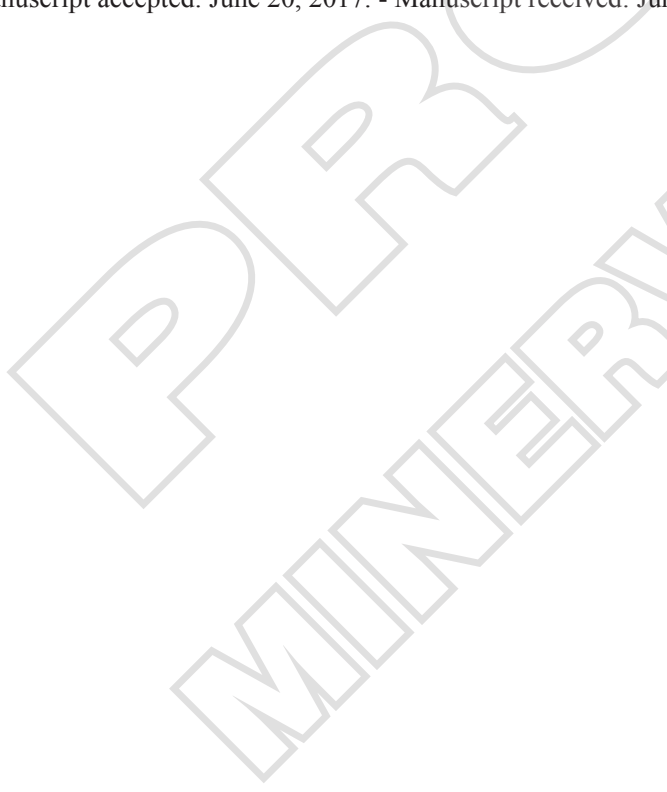

